\title{
A Full-Amplitude Nonlinear Model for RR Lyr: Pulsations, Shock Waves and $\mathrm{H} \alpha$ Peculiarities
}

\author{
Andrew Fokin \\ Institute of Astronomy, Russian Academy of Sciences \\ Ul. Pyatnitskaya 48, Moscow 109017, Russia
}

\begin{abstract}
The shock phenomena and $\mathrm{H} \alpha$ formation in the atmosphere of RR Lyrae are investigated by means of numerical simulations. The full-amplitude hydrodynamical model is generated, adopting $M=0.578 M_{\odot}, L=62 L_{\odot}, T_{\text {eff }}=7175 \mathrm{~K}$ and Population II composition. The $\mathrm{H} \alpha$ profiles are obtained by solution of the non-LTE line transfer problem for the multilevel hydrogen atom. In the course of pulsations an extended (about 16 static scales) low-density atmosphere is produced with nearly exponential density decrease. Two shocks, propagating outwards, are successively generated during one period. The shock amplitude reaches $140 \mathrm{~km} / \mathrm{s}$. No mass loss has been found. These results confirm the earlier results of S. Hill (1972). The main shock develops very high, at mass depths near $0.001 \mathrm{~g}$ per area, whereas computations for W Vir yield 100 . An analysis of the Fe I and Ti II lines shows that this difference is sufficient to explain the lack of metallic line-doubling in RR Lyrae stars. The bump on the light curve is due to a weak shock, propagating below the photosphere, which is generated with the early shock at the stage of expansion at the H-recombination front. The central intensities, Dopplerian shifts, amplitudes and phases of splitting of the computed $\mathrm{H} \alpha$ profiles agree well with the observed values (Gillet \& Crowe 1988). The lack of strong emission also fits the observations, resulting from strong scattering processes near the shock. The predicted Lyman lines show strong emission, reaching maximum at the maximum light.
\end{abstract}

